\title{
Fon Language
}

National Cancer Institute

\section{Source}

National Cancer Institute. Fon Language. NCI Thesaurus. Code C153920.

A Niger-Congo language, part of the Eastern Gbe language cluster, spoken mainly in Benin. 